\title{
SENTIDO Y DIFERENCIA UNA REFLEXIÓN SOBRE EL SENTIDO DE LA VIDA HUMANA EN LA ERA TECNOCIENTÍFICA
}

\author{
ALFREDO MARCOS \\ Universidad de Valladolid
}

\begin{abstract}
RESUMEN. La modernidad nos ha aportado potentes instrumentos, pero nos ha sumido en la ignorancia del sentido (sección 1). Para investigar sobre el sentido propongo recuperar dos máximas antiguas: «llega a ser quién eres» y "conócete a ti mismo» (sección 2). En realidad, el autoconocimiento y la autorrealización son simultáneos. Necesitaremos, pues, un conocimiento de nuestra naturaleza humana y de nuestra constitución personal. Expondremos una teoría de la naturaleza humana próxima al sentido común y a la tradición aristotélica, según la cual somos animales sociales racionales (sección 3). Esto nos da ya orientaciones de vida. Pero mi vida tiene que realizarse en función de la persona que soy ¿Podemos obtener conocimiento sobre esto? La respuesta nos viene dada a través de la noción de diferencia (sección 4). La diferencia constitutiva es única en cada persona, y es formal, luego, en principio, cognoscible. Para conocerla hace falta un arsenal muy variado de recursos, que incluye las ciencias y mucho más.
\end{abstract}

PALABRAS CLAVE: sentido, diferencia, naturaleza humana, persona.

\section{Sense and Difference. A reflection on the meaning of human life in the techno-scientific era}

ABSTRACT. Modernity has given us powerful tools, but has plunged us into a certain ignorance of the sense (section 1). To investigate on the sense or meaning I propose recover two ancient maxims: «become who you are» and «know thyself» (section 2). In fact, self-knowledge and self-realization are simultaneous. Therefore, we will need an understanding of our human nature as well as of our personal constitution. We will present a theory of human nature close to common sense and to the Aristotelian tradition, according to which we are rational social animals (section 3). This gives us some guidance for life. However, my life has to be conducted depending on the person I am. Can we get knowledge about this? The answer comes through the notion of difference (section 4). The constitutive difference is unique to each person, and is formal, then, in principle, knowable. But in order to know it, one need to meet many resources, including science and more.

KEY WORDS: sense, difference, human nature, person.

\section{INTRODUCCIÓN}

El viajero está sentado en un banco de la estación. Escribe en su libreta con aire melancólico. Acaba de dejar un autobús y está a punto de abordar otro, aunque todavía no ha decidido cuál. Hace balance y anota cómo ha cambiado él mismo, lo que ha visto, lo que ha aprendido en el viaje, a quién ha conocido, de quién se ha distanciado, lo que ha adquirido y lo que ha perdido, sorpresas, expectativas frustradas... Esta es nuestra posición histórica. Hemos viajado a través de los tiempos modernos. Fin de trayecto. Ahora estamos a punto de enrolarnos en una nueva expedición hacia no sabemos dónde. Tenemos la impresión incierta de que hacer arqueo nos ayudará a elegir mejor la nueva 
ruta. Balance de los tiempos modernos: adquisiciones, una navaja suiza; pérdidas, una brújula.

Nuestros filósofos gustan de otro lenguaje. Han llamado «racionalidad instrumental» (Habermas) a la navaja suiza y han bautizado como «sombra del nihilismo» (Gadamer) a la pérdida de la brújula. Pero con un lenguaje u otro, la cuenta es la misma: tenemos ya, por fin, los medios en pos de los cuales partimos, pero durante el viaje hemos olvidado para qué los queríamos. ¿Casualmente? Es decir, ¿el hallazgo de lo uno estará relacionado de algún modo con la pérdida de lo otro? Tal vez hemos fabricado la navaja con piezas y materiales extraídos de la brújula, en cuyo caso quedaría excluida la mera casualidad. Algo así ha sucedido, según Gadamer: el mismo método científico que nos exige la abstracción de los valores, nos otorga a cambio ingentes cantidades de información y de poder. Hemos desmontado todo un entramado de sentido para centrarnos en el conocimiento y manejo de los hechos.

Dicho todavía de otra forma, la modernidad ha tenido éxito en el plano instrumental, ha multiplicado nuestras capacidades y autonomía como no se había visto jamás antes, pero ha resultado un fracaso en cuanto al sentido, nos ha sumido en la sombra del nihilismo. Como consecuencia, en nuestros días, algunos se mueven sin pretensión ni esperanza de sentido. Asumen la acción por la acción del rebelde sin causa. Otros, en una gama que va del fanático al friki, abrazan con arbitrario ardor causas insensatas o fútiles. Y los que orientan su vida hacia objetivos tradicionalmente tenidos por sensatos, como el conocimiento, la salud, la atención a los demás, la excelencia, la felicidad o la santidad, se encuentran con serios problemas para dar razón del sentido de sus vidas, ante sí mismos y ante los otros. Todo es igual, nada es mejor. ¿Por qué habríamos de preferir el conocimiento a la ignorancia, la excelencia a la mediocridad?

Si aceptamos este balance de la modernidad, es obvio que ahora deberíamos partir hacia la cuestión del sentido de la vida, pero sin perder en este nuevo viaje las poderosas herramientas obtenidas durante los tiempos modernos, ni las de carácter tecnocientífico, ni las de tipo sociopolítico. Se trata de reparar la brújula sin estropear ahora la navaja suiza.

La cuestión del sentido de la vida se podría precisar en los siguientes términos. En primer lugar tendríamos que identificar un sentido que resulte común a todos los seres humanos. En segundo lugar, dicho sentido debería ser personal, es decir susceptible de una distinta concreción para cada uno de nosotros. Y, en tercer lugar, deberíamos ser capaces de dar razón de dicho sentido. Buscamos, pues, un sentido común, personal y razonable.

Si la cuestión del sentido no encontró buen acomodo dentro de la atmósfera moderna, tendremos que plantearla ahora en un ambiente filosófico postmoderno, en el cual no rigen ya los dogmas de la modernidad. Estos dogmas, ahora obsoletos, se formulaban, según Hans Jonas, en estos términos: 
«No hay verdades metafísicas» y "no hay camino del es al debe» ${ }^{1}$. Nuestras opciones de éxito ante la cuestión del sentido dependen, en efecto, del abandono de estos dogmas. Trataremos de buscar verdades metafísicas, a través de una ontología de la diferencia, y de obtener a partir de ellas indicaciones prácticas para organizar nuestras vidas con sentido.

\section{EN BUSCA DE LAS FUENTES DEL SENTIDO}

Desde la antigüedad clásica llegan hasta nosotros cápsulas de sabiduría que pueden ayudarnos a plantear la cuestión del sentido. Consideremos dos de ellas. En primer lugar, la conocida máxima de Píndaro: «Llega a ser el que eres» (genoi hoios essi [mathon])2. Esta fórmula la hubiese suscrito cualquier autor antiguo o medieval, no es ajena a la idea kantiana de autonomía, e incluso fue citada con aprobación en varias ocasiones por el patrono del nihilismo contemporáneo, Friedrich Nietzsche. Puestos a darle sentido a la propia vida, esta parece una recomendación universalmente aceptable: cúmplete, realízate, llega a tu plenitud. Con todo, la frase admite varias lecturas, y esta ambigüedad también nos conviene filosóficamente. La máxima indica, por una parte, que la misión de un ser humano es llegar a ser plenamente tal, o sea, un ser humano, y, por otra, que el sentido de la vida de cada cual consiste en cumplir cabalmente su ser individual concreto, en llegar a ser la persona concreta que es. Añadamos que la ubicación de la palabra mathon varía según ediciones, algunas la colocan en este verso y otras en el siguiente. Si optamos por unir la palabra en cuestión al presente verso, tendríamos esta posible traducción: «!Que llegues a ser tal cual eres, sabiéndolo!» ${ }^{3}$. En cualquier caso, esta observación filológica nos lleva a la cuestión del conocimiento. Es decir, mal puedo llegar a ser el que soy, individual o genéricamente, si no sé quién o qué soy.

Lo cual está en perfecta continuidad con la segunda cápsula de sabiduría antigua que quería traer a colación: "Conócete a ti mismo» (gnothi seauton) ${ }^{4}$. Fue inscrita en la entrada al templo de Apolo en Delfos y posiblemente empleada por Sócrates. También esconde una ventajosa ambigüedad, pues nos impele al conocimiento del ser humano en general, tanto como al conocimiento de la persona que cada cual es.

En conjunto, el mensaje es claro, si quieres dar un sentido a tu vida, un sentido común, personal y razonable, has de investigar qué es un ser humano en general y quién es la concreta persona que eres.

1 Jonas, H., El principio de responsabilidad, Herder, Barcelona, 1995, pp. 89-90

2 TEvoı oı $\sigma \varepsilon \sigma \sigma \iota[\mu \alpha \theta \omega v]$ (PíndARo, Píticas, II, 72). Agradezco a la profesora Henar Zamora, de la Universidad de Valladolid, su siempre valiosa asesoría en cuestiones filológicas.

3 O sea,"tras haberlo comprendido bien", o bien, "con plena consciencia».

$4 \quad \Gamma v \omega \theta \iota \sigma \varepsilon \alpha v \tau o v$. En latín: gnosce te ipsum. 
EL DEBATE SOBRE LA NATURALEZA HUMANA

La primera de las cuestiones, es decir la que versa sobre el ser humano en general, nos lleva al secular debate sobre la naturaleza. No voy a entrar a fondo en el mismo ${ }^{5}$, tan solo mencionaré las posiciones más comunes (negación, naturalización, artificialización) y haré una propuesta propia a partir de la cual podamos avanzar hacia una ontología de la diferencia.

Entre las teorías de la naturaleza humana, destaca la idea de que el ser humano simplemente carece de naturaleza propia, es pura libertad, se determina a sí mismo y se autoconstruye poco menos que a voluntad y desde la voluntad. Se suele citar como precedente en esta línea un texto del pensador renacentista Pico della Mirandola. En el mismo, Dios le habla a Adán con estas palabras: «No te he dado ni un lugar determinado, ni un aspecto propio, ni una prerrogativa peculiar con el fin de que poseas el lugar, el aspecto y la prerrogativa que conscientemente elijas ${ }^{6}$.

Se trata, sin duda, de una ingenua exageración, propia de un humanismo recién estrenado. El ser humano posee libertad y arbitrio, pero no está exento de condicionamientos de diverso tipo, entre los que cuentan aquellos que derivan de su propia naturaleza. Sin embargo, otros autores posteriores, desde las más diversas corrientes filosóficas, ilustración, idealismo, marxismo, conductismo, historicismo y, muy especialmente, desde el existencialismo y el nihilismo, han insistido sobre esta idea del ser humano como ajeno a cualquier naturaleza dada. Hoy día, esta perspectiva está presente en el post-humanismo de raíz nietzscheana defendido por autores como el alemán Peter Sloterdijk.

Sin naturaleza humana no habría nada en común entre el ser humano y la propia naturaleza, ni entre los humanos mismos, apresado cada cual en su incondicionada libertad y en su voluntad de poder. Este hombre sin atributos, «sin lugar, aspecto ni prerrogativa», tendría que dedicar toda su vida a decidir qué ha de hacer con la misma, desde cero, en un vacío de valores y de sentido.

Apliquemos aquí la simple sensatez basada en nuestra experiencia cotidiana: somos libres, sí, pero no de modo total e incondicionado. Y si careciésemos por completo de condicionamientos, ni siquiera podríamos ejercer nuestra libertad. Kant lo dijo en atinada metáfora: la paloma que nota la resistencia del aíre piensa que volaría mejor sin él, pero el caso es que sin esa resistencia, que condiciona y limita el vuelo, ni siquiera podría volar ${ }^{7}$. Existe una naturaleza humana que nos limita y habilita a un tiempo, que condiciona y posibilita nuestra acción.

5 Quien esté interesado puede ver: MARcos, A., «Filosofía de la naturaleza humana» en Eikasia. Revista de Filosofía. VI, 35 (noviembre 2010). http://www.revistadefilosofia.com; MARcos, A., «Nuevas perspectivas en el debate sobre la naturaleza humana», en: Pensamiento, vol. 71, núm. 269, 2015, pp. 1239-1248.

6 Della Mirandola, P., Discurso sobre la dignidad del hombre, UNAM, México, 2004, p. 14 (traducción de A. Ruiz Díaz).

7 Kant, I., Crítica de la Razón Pura, Alfaguara, Madrid, 1978, pp. $46-47$ (traducción de P. Ribas, $K v r$, Introducción, B9). 
En el otro extremo - más bien en el otro exceso- encontramos las posiciones naturalistas radicales. Según estas, el ser humano es eso, naturaleza y solo naturaleza. La pregunta por el hombre tendría, así, una sencilla respuesta: cada uno de nosotros es un organismo de la especie Homo sapiens, un primate ${ }^{8}$.

Curiosamente, las posiciones que en principio parecen contrarias producen el mismo fruto, la artificialización del ser humano, y poseen similares raíces intelectuales. La convergencia de la naturalización y de la negación se aprecia ya en Nietzsche, uno de los autores que más influyen tanto en los negadores de la naturaleza humana, como en los partidarios de su radical naturalización. Esta conexión produce también una agenda similar: trans-humanista, al estilo oxoniense, o post-humanista, al estilo continental. Desde ambas partes —negadores y naturalizadores- se propone una profunda modificación y artificialización del ser humano, «mejora» (enhancement), lo llaman. En última instancia, si la naturaleza humana es totalmente natural, entonces es técnicamente disponible, y si la naturaleza humana simplemente no existe, entonces tenemos la tarea de inventarla técnicamente. Las antropotecnias sin criterio están indicadas en ambos casos 9 .

El problema es que sin una idea normativa de naturaleza humana, es imposible hablar de mejora. Ni la negación, ni la naturalización radical de la naturaleza humana nos habilitan, por tanto, para identificar mejoras. En estas condiciones solo podríamos hablar de cambios en lo humano producidos por las antropotecnias, nunca de mejoras. Esto lo sabía muy bien Nietzsche: «La última cosa que yo pretendería — nos advierte- sería "mejorar" a la humanidad $»^{10}$.

Mi propuesta, ya en términos positivos, consiste en desarrollar una concepción de la naturaleza humana de inspiración aristotélica y próxima, por lo demás, al sentido común y a la experiencia cotidiana. En la tradición aristotélica hay una afirmación de la naturaleza humana, pero sin reducción de la misma al plano puramente natural. Se podría hablar al respecto de un naturalismo moderado. La idea de naturaleza humana propia de esta tradición tiene claras implicaciones normativas, a través de nociones como las de virtud (areté), felicidad (eudaimonía) y función (ergón) del ser humano. Puede, por tanto, ser útil para abordar nuestros actuales problemas de sentido. Hablo de desarrollar, y no meramente de recuperar, una cierta concepción de la

8 Una clara exposición de esta teoría puede verse en Mosterín, J., La naturaleza humana, Espasa, Barcelona, 2006.

9 Véase MArcos, A., «Filosofía de la naturaleza humana», en: Eikasia. Revista de Filosofía. VI, 35, noviembre 2010. http://www.revistadefilosofia.com. Para ser precisos, habría que distinguir entre un naturalismo moderado y uno radical. Según el primero - que suscribo sin reservas-, las ciencias naturales son importantes para comprender al ser humano. Para el segundo, todo lo humano es reductible a su base física y biológica. De este último es del que pretendo distanciarme.

10 Nietzsche, F., Ecce homo. Cómo se llega a ser lo que se es, Prólogo, párrafo 2. http:// www.logiamediodia.com/Mediodia/wp-content/uploads/2011/04/Friedrich-Nietzsche-EcceHomo.pdf 
naturaleza humana. Es decir, hay que poner dicha concepción a la altura de nuestros actuales conocimientos. Hoy estamos en mejor posición que cualquiera de nuestros predecesores para averiguar qué es un ser humano, y ello gracias a los recientes avances en ciencias naturales, sociales y humanas. Por eso se requiere desarrollar o traer a nuestros días una cierta concepción muy valiosa de lo humano, y no meramente recuperarla ${ }^{11}$.

Para decirlo en breve, el ser humano es, según la tradición aristotélica, un animal social racional (zoon politikon logon). El método para desarrollar esta idea ha de consistir en la apertura y exploración de cada una de estas tres cajas. Es decir, tenemos que averiguar qué incluye o qué está implícito respectivamente en nuestra condición animal, social y racional. Hay que interpretar estos tres términos a la luz de nuestros actuales conocimientos. De nuevo, estamos ante una tarea que desborda con mucho el alcance de un texto breve. Nos conformaremos, pues, con asomarnos fugazmente a cada una de estas cajas para vislumbrar algunos de los elementos presentes en su interior. Quizá resulte, a la postre, que sí tenemos lugar y prerrogativas propias.

$\mathrm{El}$ hecho de que seamos animales tiene hondas implicaciones. A veces se tiende a pasar por alto este término y damos en considerar como prácticamente sinónimas las expresiones «animal racional» y «ser racional». No lo son en absoluto. Los humanos no somos cualquier tipo de ser racional, sino muy precisamente animales. Esto nos obliga a pensar y a pensarnos desde el cuerpo, desde la experiencia del animal que somos. El viejo racionalismo desencarnado tendía a identificar al ser humano solo con la racionalidad. Hoy sabemos que esto fue un error. Muchos autores recientes, desde el propio Nietzsche a Merleau-Ponty, nos lo han hecho ver. Si, por naturaleza, somos animales, ello significa, entre otras muchas cosas, que estamos situados en un entorno natural, en un mundo (Welt) que es para nosotros entorno (Umwelt). Nos corresponde un lugar: la naturaleza como casa común. Significa también que somos vulnerables, susceptibles de daño y sufrimiento. Observemos que el hecho de ser vulnerables no nos hace menos humanos, sino que es parte de aquello en lo que consiste precisamente ser humano. Asimismo, nuestra condición animal debe hacernos recordar lo mucho que compartimos con los otros animales. En este sentido, serán de gran ayuda para iluminar la condición humana las ciencias de la vida, como la genética, la biología molecular y celular, la ecología, la etología y otras.

Nuestra condición social nos hace mutuamente dependientes y nos ubica en una determinada comunidad, la familia humana. Lo mismo que sucedía con la vulnerabilidad sucede con la dependencia, es decir, que no nos hace menos humanos, sino que es precisamente una parte de aquello en lo que consiste ser humano. Por supuesto, los hallazgos de las ciencias sociales resultan en

11 Esta labor se inscribe en un proyecto más general, consistente en la construcción de un aristotelismo postmoderno. Puede verse al respecto: Marcos, A., Postmodern Aristotle, CSP, Newcastle, 2012. 
este punto de inmensa ayuda para aquilatar la naturaleza humana. Desde el terreno de la filosofía, quizá ha sido MacIntyre quien mejor ha entendido y explicado en los últimos tiempos este aspecto de lo humano. Él ha sabido desarrollar la antigua idea aristotélica del ser humano como animal político hasta su formulación contemporánea como animal dependiente. Hasta para ser autónomos dependemos de los demás, y al servicio de los demás hemos de poner nuestra autonomía ${ }^{12}$.

Con esta observación ya estamos abriendo la tapa de la tercera caja, la que llamamos racionalidad. Somos racionales, sí. Esto nos ubica en una nueva esfera espiritual. Incluye nuestra capacidad de pensar y de pensarnos, de reflexionar, de contemplar y de ponderar las razones para hacer y creer. Porque somos racionales pedimos y damos razón, buscamos explicaciones y causas, incluidas las más radicales y últimas, deliberamos, decidimos voluntariamente en un sentido u otro, valoramos la verdad, el bien y la belleza. Entiendo aquí lo racional en un sentido amplio y contemporáneo, que incluye e integra la inteligencia emocional, las aportaciones de la intuición, y en general la sensatez. No cabe duda de que las ciencias humanas, y otras perspectivas, como las que podemos obtener de las artes o de la religión, aportan luz en la tarea de perfilar estas características de lo humano. Gracias al aspecto racional de la condición humana nos constituimos como sujetos autónomos, podemos darnos a nosotros mismos las normas y criterios, y aceptar o no de manera lúcida y libre aquellas orientaciones que recibimos de fuera. Nuestra capacidad de autonomía, tal y como lo vio Kant, arraiga en esta zona de lo humano.

En resumen, esta es, pues, nuestra naturaleza: somos animales sociales racionales; en virtud de lo cual estas son nuestras prerrogativas o rasgos, nuestras diferencias: somos vulnerables, dependientes y autónomos; nos corresponden como lugares propios en los que desarrollar nuestra vida el entorno natural, la familia humana y la esfera del espíritu.

Lo interesante del caso es que estas tres dimensiones de lo humano, a las cuales nos hemos asomado tan apresuradamente, no son reductibles entre sí ni están meramente yuxtapuestas. Su relación mutua viene mejor descrita por el término diferenciación: cada una de ellas impregna completamente a las otras dos, las diferencia. Nuestra inteligencia es sentiente, nuestra forma de percibir ya viene modulada por nuestro pensamiento, nuestra racionalidad es social y dialógica, no se construye sino en comunicación con los otros, nuestras funciones animales las llevamos a cabo de modo cultural, nuestra autonomía, como decíamos más arriba, está al servicio de los dependientes y dependemos de los demás para llegar a construirla...

12 MacIntyre, A., Animales racionales y dependientes, Paidós, Barcelona, 2001, p. 10 [título original: Dependent Rational Animals, Carus Publishing Company, 1999]; MARcos, A., «Antropología de la dependencia», en Muñoz, A. (ed.), El cuidado de las personas dependientes ante la crisis del estado de bienestar, Tirant Lo Blanch, Valencia, 2013, pp. 21-34; MARcos, A., «Dependientes y racionales: la familia humana», en: Cuadernos de Bioética, XXIII, 2012 / 1 pp. 83-95. 
Tomemos como somera ilustración de este hecho el caso del amor. Los griegos fueron tan sutiles como para distinguir tres tipos: eros, philía y agapé. El primero tiende a la posesión o dominación de algún otro, el segundo es un tipo de amor simétrico, el tercero impulsa a la donación de uno mismo. Podríamos identificarlos respectivamente con el deseo sexual, la amistad y el altruismo o caridad. El primero parece radicarse en nuestra condición animal, el segundo en la social y el tercero en la intelectual (en cuanto fruto de la razón práctica). La pulsión sexual se da en casi todo el mundo animal, y en parte del mismo se pueden encontrar también ejemplos de apoyo mutuo. Quizá pensemos, pues, que lo propio y distintivamente humano es el altruismo genuino, el único no reductible al egoísmo genético. Pero no es así de simple, pues obviamente la vida humana incluye también amistad y deseo sexual, solo que ambos con frecuencia quedan integrados, impregnados o modulados —diferenciados, digamos- por el genuino altruismo. Y cuando no resulta así, cuando la amistad es pura mutualidad y el sexo puro afán de posesión y dominio, los tenemos por poco humanos, del mismo modo que tendríamos por escasamente humano un amor de absoluta y total donación, sin ni siquiera la remota esperanza de una mínima correspondencia, o un amor totalmente desencarnado - platónico, se suele decir- sin el más mínimo matiz de corporalidad. Dicho de otro modo, el amor humano concreto es una realidad compleja, en la que solo por un ejercicio de análisis descubrimos sus diversos aspectos o dimensiones. Pero aun después del análisis, hemos de recordar que, en realidad, se trata de algo único.

Si vamos más al fondo de la cuestión, nos damos cuenta de que hemos obtenido cierta información sobre lo humano, cierta lucidez, mediante análisis y abstracción. Dividimos conceptualmente lo que físicamente es uno, y consideramos por separado en nuestra mente, de modo abstracto, cada uno de los aspectos que hemos distinguido. Se trata de una aproximación a la realidad mediante operaciones del logos, que al mismo tiempo nos han alejado del plano real, físico. Esta distinción entre lo lógico y lo físico tiene antiguas raíces en la filosofía aristotélica y ha sido precisada más recientemente por Zubiri. Según este: "Físico es el vocablo originario y antiguo para designar algo que no es meramente conceptivo sino real» ${ }^{13}$. Lo físico, en este sentido, es lo real, lo que es independiente del concepto, del logos. Incluso Dios sería un ser físico en este sentido zubiriano, es decir, con existencia plena e independiente de nuestros conceptos, análisis y abstracciones. Cada persona, vistas así las cosas, es también un ser físico, real, sustantivo.

Para volver a lo real desde lo conceptual, a la sustantividad que es cada persona, hemos de tener siempre presente que lo humano se da de manera integral, unitaria, indivisible en cada uno de nosotros. ¿Qué tipo de ontología podría hacer justicia, tanto al análisis conceptual que hemos hecho de lo humano, como a la realidad integral única que es cada persona? Dicho de otro

13 Zubiri, X., Sobre la esencia, Sociedad de Estudios y Publicaciones, Madrid, 1980 [primera ed. 1962], p. 22. 
modo, ¿cómo podríamos conectar el concepto de naturaleza humana con la realidad sustantiva que es cada persona? La cuestión es clave, pues en esta conexión nos jugamos el autoconocimiento y, con ello, el sentido de la vida.

\section{ONTOLOGÍA DE LA DIFERENCIA}

Mantengamos el reto: conócete a ti mismo para llegar a ser quien eres. Pero conocer la naturaleza humana no es todavía saber quién soy. Obviamente, saber qué soy aporta mucha luz sobre el problema de quién soy, pero me deja indefectiblemente a cierta distancia de la meta. No puedo orientar mi vida sin saberme animal social racional, pero este saber no es suficiente, necesito conocer también la persona concreta que soy.

Estamos ante un doble problema. Por un lado, nos preguntamos por el posible conocimiento de lo individualidad concreto. No soy simplemente un animal, sino este animal, no soy simplemente social, sino que pertenezco en concreto a esta familia, a esta población, a este pueblo o polis, no soy simplemente un ser racional, sino que soy exactamente este ser racional. Por otro lado, está la cuestión de la unidad: los tres rasgos o diferencias han de integrarse de algún modo si quiero dar cuenta del ser sustantivo que soy. Los dos aspectos de este problema apuntan hacia el mismo concepto, ajeno ya al universo griego, y más propio de la filosofía medieval y de la tradición juedeocristiana, el de persona. Decir que soy este animal social y racional concreto es tanto como decir que soy esta persona; así el qué abstracto se concreta en un quién. Además, soy una persona precisamente, y no más. En la persona, los tres rasgos o diferencias de la naturaleza humana se unifican e integran al tiempo que se concretan ${ }^{14}$.

Mi hipótesis es que las dos cuestiones de que consta el aterrizaje de la naturaleza humana en la persona concreta se pueden afrontar gracias a la noción de diferencia. Y esto nos coloca ya en la atmósfera típica de la filosofía postmoderna. La crítica heideggeriana al olvido de la diferencia, así como su reivindicación de esta noción, encontró eco inmediato en otros pensadores contemporáneos, como Deleuze, Lyotard o Derrida ${ }^{15}$. Y con ello nos viene sugerida una nueva dificultad quizás insalvable, la de la inteligibilidad de la

14 Hay que aclarar que esta integración y concreción no es igual a una síntesis. De hecho, ningún organismo llega a la existencia mediante síntesis o composición de partes preexistentes, sino mediante un proceso de diferenciación. Esto debería hacernos pensar, dicho sea de paso, sobre el tipo de tecnociencia que es la que hoy llamamos, en términos que parecen entre sí contradictorios, biología sintética.

15 Heidegger, M., Identidad y diferencia, Anthropos, Barcelona, 1988 [ed. bilingüe a cargo de A. Leyte y H. Cortés, ed. original en alemán, Identität und Differenz, Klett-Cotta, Stuttgart, 1955-57]; Deleuze, G., Diferencia y repetición, Júcar, Madrid, 1988 [trad. A. Cardín, ed. original en francés Différence et répétition, P.U.F., París, 1968]; LyotaRD, J. F., La diferencia, Gedisa, Barcelona, 1988 [trad. A. L. Bixio, ed. original en francés, Le différend, Minuit, París, 1983]; Derrida, J., La escritura y la diferencia, Anthropos, Barcelona, 2012 [trad. P. Peñalver, ed. original en francés, L'Écriture et la différence, Seuil, París, 1967 ]. 
diferencia. Recordemos la vieja tradición parmenídea conforme a la cual solo la identidad es realmente inteligible. Si tratamos de captar el quién, la persona, a través de la noción de diferencia, quizá nos condenamos a no entender a la persona, a no saber quién es cada cual y, en consecuencia, a la pérdida del sentido. Con todo, la cuestión es demasiado importante como para dejarla pasar sin una exploración más detenida: se trata de saber si es posible o no establecer razonablemente en plena postmodernidad el sentido de una vida humana.

Permítaseme, pues, trabajar sobre la noción de diferencia. Quizá, una vez reconsiderada, podamos emplearla para transitar desde la naturaleza humana, hasta la persona concreta, desde el qué hasta el quién. Quizá podamos hacerlo sin poner en riesgo la unidad de la persona, unidad aparentemente amenazada por una definición formulada en términos de tres diferencias. E incluso es posible que nuestro trabajo sobre la noción de diferencia acabe por mostrar que, en el fondo, y a su manera, también la diferencia, y no solo la identidad, resulta inteligible.

\section{Diferencia constitutiva y diferencia comparativa}

Abordaré la noción de diferencia desde la perspectiva de la biología de Aristóteles, donde tal noción resulta clave. Cuando leemos a Aristóteles desde su biología, como se ha empezado a hacer en las últimas décadas, su pensamiento conecta de modo muy natural con el de autores contemporáneos como Zubiri o los filósofos de la diferencia (y muy especialmente Deleuze). Los textos biológicos de Aristóteles denominan diferencia (diaphorá) a cada rasgo de un ser vivo. El viviparismo, la condición de herbívoro, la posesión de alas o de vesícula biliar son diferencias. De hecho, la biología del pensador griego está estructurada según diferencias, no según especies. Por ejemplo, es posible encontrar en líneas muy próximas entre sí referencias al delfín y al caballo por el hecho de que ambos son vivíparos. O bien: se interesa por el topo porque en él se dan dos diferencias, ceguera y viviparismo, que raramente aparecen juntas. Trata, en suma, sobre la condición de vivíparo, de ciego o de herbívoro, no sobre tal o cual especie. Podríamos inferir que en el caso del ser humano sucede algo parecido, lo importante no será la especie, sino las diferencias.

Pero la noción de diferencia tiene, en Aristóteles, al menos dos significados que conviene distinguir. La diferencia puede ser entendida en un sentido lógico, como rasgo que diferencia, distingue, separa, una clase de otras, o bien en su sentido físico. En este segundo sentido se trata del rasgo en tanto que constitutivo de un ser vivo concreto. Para ambas acepciones podemos hallar usos en español. Según la primera, decimos que dos entidades son diferentes en tal o cual característica. Aquí la diferencia compara. Conforme a la segunda, hablamos del proceso de diferenciación de un viviente, que es tanto como su ontogénesis, la génesis de lo heterogéneo a partir de lo homogéneo y, con ello, la constitución de la propia entidad. Aquí la diferencia constituye.

Aristóteles hereda de Platón la primera acepción de la diferencia, y la mantiene. Pero añade la otra, más propiamente biológica. Nos referimos al 
segundo de los sentidos mencionados. En este mismo sentido se utiliza hoy el concepto de diferenciación en embriología, para señalar el proceso mediante el cual aparecen células y tejidos más diferenciados a partir de otros que lo están menos. El primer sentido es más clasificatorio, comparativo y estático, mientras que el segundo es más dinámico y constitutivo. El primero es principalmente lógico y el segundo físico. Bien se podría decir, pues, que las diferencias comparativas son, en realidad, un subproducto de las constitutivas.

En la contemporánea filosofía de la diferencia podemos encontrar también una idea de diferencia constitutiva muy próxima a la de Aristóteles. El pensamiento de Deleuze, por ejemplo, se caracteriza por el deliberado intento de inversión de las nociones de identidad y diferencia. Tradicionalmente la diferencia se tomaba como algo secundario y derivado de la identidad. Para que puedan existir diferencias — se suponía-, antes deben existir entidades idénticas cada una a sí misma entre las cuales establecer las diferencias. Para Deleuze, a la inversa, son las diferencias las que generan identidades. De hecho, la identidad de una entidad vendría dada por una serie de diferencias que, al mismo tiempo, constituyen internamente la entidad (diferencias internas) y, secundariamente, la distinguen de otras entidades. De este modo, la relación entre lo general y lo concreto no sería ya una relación lógica de subsunción, sino una relación física de diferenciación. Por lo tanto, si queremos llegar a conocer a la persona que somos, no podemos conformarnos solo con lo general, sino que tenemos que ir a lo primario y constitutivo, a la diferencia.

Las dos acepciones apuntan también a dos modos distintos de conocer. Tomada la diferencia en el plano lógico, el conocimiento resultante es clasificatorio y la definición de un objeto se produce por comparación con otros y por su ubicación en un determinado dominio conceptual. Mientras que la diferencia en su acepción física nos orienta hacia la organización propia del objeto, hacia su constitución interna y sus relaciones reales con otros, es decir, hacia su forma individual, hacia su esencia.

Pudiera parecer extraña la alusión a una forma individual —que es forma de vida en el caso de los vivientes-, y más extraño todavía que identifiquemos el viviente individual con su esencia. Pero, en mi opinión, hay buenos argumentos a favor de esta identificación. Zubiri, por ejemplo, en Sobre la esencia, aporta algunos. Y el propio Aristóteles, habida cuenta de la reciente investigación sobre su biología, puede ser interpretado perfectamente como defensor de una forma cuantitativa y cualitativamente individual, identificable en cada ser precisamente con su esencia ${ }^{16}$. A este respecto, Theodor Scaltsas ha escrito: «La esencia no puede pertenecer al sujeto, tiene que ser el propio sujeto» ${ }^{17}$.

16 Sigo en esta interpretación a autores contemporáneos como Pierre Pellegrin y David Balme. He argumentado largamente a favor de la misma en diversos lugares: MARcos, A., Aristóteles y otros animales, PPU, Barcelona, 1996; MARcos, A., Postmodern Aristotle, CSP, Newcastle, 2012.

17 Scaltsas, Th., Substances \& Universals in Aristotle's Metaphysics, Cornell University Press, Ithaca, NY, 1994, p. 3 
Y el mismo Aristóteles se pregunta: «¿Qué impide que ciertas realidades se identifiquen ya con su esencia, dado que la esencia es sustancia?» ${ }^{18}$.

En suma, al autoconocimiento se accede mediante el conocimiento de las diferencias, y la autorrealización consistirá precisamente en un proceso de diferenciación. Nos sigue preocupando, no obstante, la cuestión de la unidad de la persona, pues estamos hablando todavía de diferencias, así, en plural. ¿Será posible la integración de todas ellas en una sola?

\section{La unidad de las diferencias en la última diferencia}

La diferencia constitutiva solo puede ser una y única, pues constituye a un ser uno y único. Es más, la diferencia constitutiva, en realidad, se identifica con la propia sustancia que constituye. En el caso que nos ocupa, el del ser humano, la diferencia constitutiva sería la propia persona. En esta dirección apuntan varios textos del tratado Sobre el Alma y del resto de la obra biológica de Aristóteles. Permítaseme considerar uno de ellos, tal vez el más significativo. Hay un pasaje del tratado Sobre las partes de los animales en el que Aristóteles afirma que «la diferencia es la forma en la materia» ${ }^{19}$. Para algunos podría resultar extraña esta afirmación ${ }^{20}$. Y lo es si la leemos desde el punto de vista lógico, pero no si lo hacemos desde el punto de vista físico.

Desde el punto de vista lógico, la especie es el resultado de añadir al género la diferencia. O sea, da la impresión de que la especie está más cerca de la materia, y que la materia es el principio de individuación de la especie. Pero desde el punto de vista físico las cosas cambian, es la diferencia la que está más cerca de la materia. La diferencia es la forma en la materia, la forma en la entidad individual concreta, y, en realidad, esta misma entidad ${ }^{21}$, pues son muchos los textos en los que Aristóteles reafirma la unidad de materia y forma ${ }^{22}$. Es la forma, entendida como diferencia, la que juega aquí como principio de individuación de una materia indiferenciada o genérica. De modo que la diferencia constitutiva no es una forma en abstracto, sino la forma en la materia, el viviente concreto, si hablamos de seres vivos, la persona, si de seres humanos se trata. Esta lectura no es excepcional, sino que enlaza de modo muy natural con otros textos situados en el libro II del tratado Sobre el alma y en Metafísica, libros VII y VIII.

18 ARIstóteles, Metafísica, $1031 \mathrm{~b} 31$ y ss.

19 ARistóteles, Sobre las partes de los animales, 643a 24.

20 Incluso se da el caso de editores y traductores de los textos de Aristóteles que han tratado de enmendarla. Sin embargo, figura tal cual en todos los manuscritos menos en uno. Véase al respecto Bartolomé, R. y Marcos, A., Aristóteles: Obra biológica, Luarna, Madrid, 2010, p. 113, n. 166, en www.fyl.uva.es/ wfilosof/webMarcos/textos/Textos_2013/Aristoteles_ Obra_biologica.pdf.

${ }^{21}$ Véase al respecto Inciarte, F., El reto del positivismo lógico, Rialp, Madrid, 1974, p. 276.

22 Aristóteles, Metafísica VIII 6; Sobre el alma II 1; Sobre las partes de los animales I; véase también Marcos, A., Aristóteles y otros animales, PPU, Barcelona, pp. 140-142. 
Está claro, pues, que desde el punto de vista físico la diferencia constitutiva es única y se identifica con la cosa misma; en el caso de los seres humanos, con la persona. Ahora bien, se nos plantea el problema de la relación entre lo físico y lo lógico. Es decir, ¿podríamos de algún modo captar esta diferencia desde nuestros conceptos, podríamos definir a cada persona, conocer lo individual? ¿Podríamos integrar los distintos rasgos o diferencias propias de la naturaleza humana en una sola y última diferencia lógica que se corresponda con la diferencia física constituyente? En ese caso, la diferencia última sería a un tiempo la sustancia y la definición de la cosa ${ }^{23}$. Estamos ante el problema de la inteligibilidad de la diferencia o, si se quiere, de lo individual concreto, que para el caso que nos ocupa es tanto como hablar de la inteligibilidad de la persona. Pasemos ahora a considerar esta cuestión.

\section{La inteligibilidad de la última diferencia}

Si la separación entre logos y physis fuese insalvable, tendríamos que renunciar al autoconocimiento y a la justificación del sentido. Aristóteles se embarca por dos veces en el intento de cerrar la brecha entre las diferencias lógicas y la diferencia física. Lo hace, en primer lugar, mediante una reforma de la teoría de la definición. Y fracasa. Emprende después una nueva singladura en busca de otra forma conocimiento (alle gnosis ${ }^{24}$ ), esta vez a través de la filosofía práctica y de una nueva constelación de ideas, entre las que se encuentran las de analogía, metáfora, semejanza, prudencia y verdad práctica. La filosofía actual de la diferencia ha tomado nota, y con razón, del fracaso del primer recorrido, el de la definición y el logos unívoco, pero no ha sabido valorar suficientemente el potencial del segundo. Podríamos, en mi opinión, disipar la sombra del nihilismo actualizando las sugerencias que Aristóteles nos brinda en esta su segunda singladura.

La reforma del aparato lógico de la definición es llevada a cabo por el pensador griego por los siguientes pasos. Primero reduce todos los géneros que puedan figurar en una definición a uno: «En la definición no entra otra cosa que el género denominado primero y las diferencias» ${ }^{25}$. A continuación reduce el género a la especie: «El género no existe en absoluto aparte de las especies del género, o si existe es como materia ${ }^{26}$. En un tercer paso reduce la especie a las diferencias: "Es evidente que la definición es el enunciado constituido a partir de las diferencias ${ }^{27}$. Y el cuarto paso reduce todas las diferencias a la última: "Es evidente que la diferencia última será la sustancia y la definición de la $\cos a{ }^{28}$. Luego, si se pudiese hacer bien el proceso, es decir, dividiendo

\footnotetext{
23 Metafísica, 1038a 19-20.

24 Sobre la generación de los animales, 742b 32.

Metafísica, 1037b 30 - 1038a 4.

Metafísica, 1038a 5-8.

Metafísica, 1038a 8-9.

Metafísica, 1038a 19-20.
} 
por la diferencia de la diferencia ${ }^{29}$, entonces, toda la definición y, lo que es más, la sustancia misma, estaría contenida en la última diferencia ${ }^{30}$, que sería a un tiempo lógica y física.

Pero en el tratado Sobre las partes de los animales ${ }^{31}$ salen ya a flote numerosos problemas a la hora de establecer conexiones entre la physis y el logos por la vía de la definición. La definición que conduce a la diferencia última, dividiendo por la diferencia de la diferencia, no parece factible, de modo que a la hora de investigar sobre los animales, hay que proceder mediante varias series de diferencias, por así decirlo, paralelas, que no confluyen en una última y única. Así, lo más recomendable es proceder como hace el saber común, poniendo juntos aquellos individuos que comparten una cierta constelación de diferencias, que no podemos ya comprimir en una única ${ }^{32}$. Aquí parece que finaliza el idilio entre la physis y el logos. La mayor proximidad entre lo uno y lo otro la hallaríamos bajo fórmulas como «Sócrates es racional» (su diferencia específica), que expresa más sobre Sócrates que "Sócrates es hombre» (su especie $)^{33}$. Aun así, no pone pie en la individualidad de la persona, ni expresa correctamente la integración de los distintos aspectos biológicos, sociales y espirituales de la misma. No es extraño que el propio Aristóteles muestre en algunos pasajes una clara desconfianza respecto del logos unívoco y de las virtualidades de la definición ${ }^{34}$.

¿En qué consiste, pues, esa otra forma de conocimiento (alle gnosis) que nos llevaría más cerca de la persona? En primer lugar, para que este conocimiento sea meramente posible, es importante reparar en la naturaleza formal de la diferencia como principio de individuación. Solo si reconocemos este aspecto formal a los individuos pueden estos resultar inteligibles. Se puede interpretar la filosofía aristotélica de muchos modos, e históricamente se hecho. Pero en nuestros días, a partir de la lectura atenta de los textos biológicos, está emergiendo una lectura según la cual la forma es individual. Lo es cuantitativamente en todos y cada uno de los seres, y lo es cualitativamente de manera gradual. Es decir, la diferenciación cualitativa individual admite grados; así, una abeja, cuya plasticidad comportamental es escasa, pues su conducta está genéticamente regulada de modo rígido, presenta menores diferencias respecto de otras que un delfín, cuya capacidad de aprendizaje es considerablemente mayor, respecto de otros. Por ello, en ciertos casos, lo que aprendemos sobre la especie puede casi agotar lo que podemos aprender

29 Metafísica, 1038a 9; Sobre las partes de los animales, 642b 5 - 644a 12.

30 Un buen relato de este proceso de reducción, que he seguido aquí, aparece en INCIARTE, F., El reto del positivismo lógico, Rialp, Madrid, 1974, pp. 251-252.

31 Sobre las partes de los animales, 643b 10 y ss.

32 Por ejemplo, se entiende que son aves los sanguíneos, ovíparos, alados, plumíferos, con huesos huecos, con pico y sin dientes. Cada una de estas diferencias puede ser el extremo de una serie distinta y ninguna de ellas tiene por qué incluir a todas las demás.

33 Sobre las partes de los animales, 645b 13-22; Sobre el alma, 402b 10-16, 415a 16-20.

34 Refutaciones sofísticas, 165a 5-14. 
acerca de cada individuo. Pero en otros casos, una vez conocidos los rasgos de la especie, quedará aún mucho que aprender acerca de cada individuo. Y el caso humano es extremo en este sentido.

Por más que aprendamos sobre la naturaleza humana, quedará todavía una enorme cantidad de conocimiento por obtener acerca de cada persona concreta. Para esta tarea serán imprescindibles las ciencias, en una cierta interpretación analógica de sus conceptos, pero también las artes, con su capacidad de creación de metáforas, así como otras fuentes de conocimiento, tales que la religión, el diálogo y la comunicación con los otros, la meditación y reflexión, la propia praxis y la experiencia vital cotidiana, interpretado todo ello bajo la modulación de la prudencia.

Parece que mediante una sensata combinación de todas estas fuentes de conocimiento podemos poner pie, o casi, en lo individual concreto. Pero ahora deberíamos preguntarnos en qué medida estas fuentes de conocimiento responden ante el tribunal de la verdad. Hasta hace poco, de un modo u otro, se mantuvo la distinción platónica entre ciencia (episteme) y opinión (doxa), como dos modos o niveles distintos del saber, dotado de certeza el primero y no el segundo. Algunos autores postmodernos han enrasado esta distinción al interpretar en clave relativista toda forma de conocimiento, incluida la ciencia. Otros pensadores, como Karl Popper, han optado por distinguir cuidadosamente entre verdad y certeza, para reconocer a continuación que incluso en ciencia hemos de convivir con la incertidumbre, pero que ello no impide que toda fuente de conocimiento, si se pretende legítima, haya de responder ante el tribunal de la verdad. A partir de aquí, nuestra pregunta podría formularse en estos términos: ¿El conocimiento sobre cada individuo concreto, procedente de una pluralidad de fuentes, en qué modo responde ante el tribunal de la verdad?

La pregunta es importante, para empezar porque el andamiaje conceptual de la ciencia lo construimos a partir de un cierto conocimiento de los individuos concretos, por semejanza entre diferencias. Así elaboramos los conceptos, las leyes y las clasificaciones: a partir de las semejanzas entre diferencias (semejanzas que descubrimos creativamente ${ }^{35}$ ). Por supuesto, también es importante porque la verdad en el conocimiento de la persona es imprescindible para establecer de modo razonable y sin relativismo el sentido de su vida.

\section{Verdad práctica y autorrealización}

Pues bien, el tipo de verdad que podemos pedir al conocimiento de lo individual es la verdad práctica. Según Aristóteles: «el bien de la parte

35 Sobre la importancia epistémica de la semejanza puede verse MARcos, A., Postmodern Aristotle, Newcastle, 2012, cap. 6; Marcos, A., "Semejanza», en: Estudios Filosóficos, vol. LX, $n^{\circ} 173,2011$, pp. 119-136. "We can only say "the same" if we think difference», afirma Heidegger en "... Poetically Man Dwells...», in Heidegger, M., Poetry, Language, Thought, Harper, New York, 1971, pp. 213-229; disponible en: http://timothyquigley.net/cont/heideggerpmd.pdf. 
intelectual pero práctica es la verdad que está de acuerdo con el deseo recto [...] esta clase de entendimiento y de verdad es práctica ${ }^{36}$. Destaquemos algunas de las características de la verdad práctica.

En primer lugar, sabemos que consiste en la concordancia entre deseo e intelecto, pero sin que el uno domine sobre el otro, sin que ninguno de los dos sufra violencia para adecuarse al otro, pues en ese momento el ser humano, que es «deseo inteligente e inteligencia deseosa» ${ }^{37}$, se estaría haciendo traición a sí mismo, estaría dejando se ser auténtico, verdadero. Obsérvese la fórmula gramatical elegida por Aristóteles para referirse aquí al ser humano. No emplea la yuxtaposición, sino la adjetivación de un rasgo mediante el otro, es decir la diferencia en la diferencia. Y se cuida muy bien de evitar la jerarquización, al poner cada uno de los dos términos tanto en la función de nombre como en la de adjetivo. La verdad práctica consiste en la integración de estas dos diferencias en un punto intermedio y mejor, que no está previamente dado en ninguna de ellas, sino que debe ser hecho. Y al mismo tiempo que es hecho es descubierto. Se da por y en la acción.

En segundo lugar, se puede decir con toda propiedad que existe un tipo de verdad que no se concibe como un acuerdo abstracto, sino que se hace, se realiza o, para ser más precisos, se actualiza mediante la acción de un sujeto. Dicha verdad puede ser entendida, pues, como la actualización de una potencia: en la medida en que tal potencia era real, la verdad práctica es objetiva, es genuina verdad; en la medida en que la actualización de la misma requiere acción humana, es creativa, es práctica.

Y, por último, no hay una regla automática para la creación ni para el reconocimiento de este tipo de verdad; sin embargo, la arbitrariedad y el relativismo están excluidos, se trata de un ejercicio «determinado por la razón y por aquélla regla por la cual decidiría el hombre prudente» ${ }^{38}$.

Así pues, el concepto de verdad práctica está pensado en las dimensiones ética, epistémica y antropológica, como la realización de cada ser humano por acuerdo de intelecto y deseo, como un proceso de actualización de capacidades humanas que se da en la acción y bajo la guía de la prudencia.

Lo importante aquí es que el modelo de la verdad práctica nos permite cerrar la brecha entre la physis y el logos tanto como sea posible. Ciertas características o diferencias se aprenden, se constituyen y se integran a un mismo tiempo, dando como resultado la persona misma. Este modelo, que Aristóteles aplica a dos de nuestros rasgos, la condición desiderativa/animal y la intelectual/ racional, tal vez pueda ampliarse hasta abarcar las tres diferencias propias de la naturaleza humana. Para dar el primer paso en esta dirección, quizá baste con recordar que nuestra inteligencia es también social, es decir comunicativa y dialógica.

36 Ética a Nicómaco, 1139 a 26 y ss.

37 Ética a Nicómaco, 1139b 6.

38 Ética a Nicómaco, 1106b 36 y ss. 
Verdad práctica significa, pues, concordancia entre deseo e intelecto, y constitución de la propia persona. Cuando se da la concordancia entre el deseo y la inteligencia el sujeto se va constituyendo, diferenciando y mejorando a sí mismo. De manera que la verdad práctica no consiste tan sólo en el acuerdo entre dos rasgos humanos, sino que tiene una dimensión constitutiva: «Con razón se dice - escribe Aristóteles- que realizando acciones justas se hace uno justo [...] y sin hacerlas ninguno tiene la menor posibilidad de llegar a ser bueno» ${ }^{39}$. Es decir, para conocernos con verdad ("conócete a ti mismo»), tenemos que hacernos («llega a ser el que eres»). «Lo que hay que hacer después de haber aprendido -insiste Aristóteles- lo aprendemos haciéndolo» ${ }^{40}$.

Obsérvese que aquí hemos invertido la relación de orden entre las dos máximas. Empezábamos diciendo que para cumplir con la máxima de Píndaro uno tiene que conocerse a sí mismo. Descubrimos ahora que, para conocerse, uno tiene que realizarse. No se trata de un círculo vicioso sin posible entrada ni comienzo. Sino de un círculo virtuoso en el que simultáneamente uno se conoce por autorrealización y mediante ese conocimiento aprende lo que tiene que realizar. Entre la dimensión ética («llega a ser el que eres») y la epistémica («conócete a ti mismo») de la verdad práctica existe una mutua implicación exigida por la propia antropología aristotélica. Llega a ser el que eres, es decir, conócete a ti mismo. Conócete a ti mismo, es decir, realízate.

Uno se da cuenta de que tiene talento para la música haciendo música, aprendemos sobre nuestra capacidad para el deporte ejercitándola, en la acción llegamos a captar nuestras dotes para la concentración o para el trabajo prolongado y duro, para la observación, para la atención al detalle o bien para la creatividad, para la compasión o el cuidado, para la gestión o el liderazgo, para el cálculo o la abstracción, para la reflexión o la meditación, para el humor o el buen consejo, descubrimos, en fin, la textura propia, concreta e irrepetible de cada persona. Lo que después de haber aprendido hemos de hacer, lo aprendemos haciéndolo.

En esta búsqueda del autoconocimiento y de la realización de cada persona, tienen un papel fundamental los demás. De hecho, la puerta de entrada al círculo virtuoso es la paideia $^{41}$. Recordemos, nuestra inteligencia no solo es deseosa, sino también social. Nuestro autoconocimiento y realización dependen críticamente de aquellos que saben ver las capacidades y posibilidades, de aquellos que aconsejan y orientan, que dan ejemplo, que educan, entrenan, cuidan, sanan o forman. También de nuestros pares en la aventura de la vida, cuya conversación nos resulta de tanta ayuda para autodescubrirnos. Y junto con ello, las distintas modalidades del arte y de la ficción nos aportan visiones de la vida humana a través de las cuales de un modo u otro podemos vislumbrar la propia. Y aun habría que sumar a todo esto otras fuentes de conocimiento; en

39 Ética a Nicómaco, 1105b 10 y ss.

40 Ética a Nicómaco, 1103a 32 y ss.

41 Véase Marcos, A., "Aprender haciendo: paideia y phronesis en Aristóteles», en: Educação (Brasil), vol. 34, nº 1, 2011, pp. 13-24. 
palabras de Gadamer: «Uno no puede ignorar tal "conocimiento” en cualquier forma en que se exprese: en la sabiduría religiosa o proverbial, en obras de arte o en pensamiento filosófico» ${ }^{42}$.

Se podría objetar que también mediante el ejercicio asiduo del crimen, uno puede descubrir en sí mismo ciertas dotes y capacidades criminales. En este caso, ¿debería uno esforzarse en el perfeccionamiento de estas dotes para tener una vida cumplida? Desde luego, si la coherencia con lo dicho nos obligase a responder afirmativamente, estaríamos ante una especie de reducción al absurdo de toda la posición que hemos ido edificando. Pero no hay por qué responder afirmativamente, pues el ejercicio universal del crimen sería obviamente contrario a nuestra naturaleza social, e incluso peligroso para nuestra condición animal, mientras que reservar solo para uno mismo el privilegio del crimen está en contra de nuestra condición racional, como argumenta Kant.

Es decir, el hecho de que el autoconocimiento y la autorrealización respondan ante el tribunal de la verdad implica que no toda biografía es genuina autorrealización. El relativismo, insisto, queda excluido. Prueba de ello es que podemos decir, cada uno de sí mismo y de los demás, de modo falible pero no injustificado, cuándo estamos ante una vida realizada (praxis teleia) y cuándo ante una vida fracasada, inauténtica, errónea (en el doble sentido, ético y epistémico). Para empezar, cualquier tipo de biografía que no atienda a la naturaleza humana será una suerte de autofalsificación, pues somos humanos. Esta clave es la que nos ha permitido excluir el estilo de vida criminal como una genuina vía de autorrealización. Dicho de otro modo, la naturaleza humana tiene valor normativo para nuestras vidas. Afirma Aristóteles que «lo que es propio de cada uno por naturaleza es lo mejor y lo más agradable para cada uno» ${ }^{43}$.

De las notas propias de nuestra naturaleza, parece que Aristóteles señala la racionalidad como la más excelente e incluso divina, y recomienda «en la medida de lo posible, inmortalizarnos y hacer todo esfuerzo para vivir de acuerdo con lo más excelente que hay en nosotros $»^{44}$. Sin embargo, en un pasaje aledaño, nos recuerda que "siendo humano, el hombre contemplativo necesitará del bienestar externo, ya que nuestra naturaleza no se basta a sí misma para la contemplación, sino que necesita de la salud corporal, del alimento y de los demás cuidados» ${ }^{45}$. En definitiva, no podemos olvidar nuestros aspectos animales y sociales, y tampoco traicionar a la parte más divina que hay en nosotros, sino buscar una integración armónica y con vistas a la excelencia de todo ello. Esa es la posición de lo propiamente humano.

Por otro lado, y aun dentro de los márgenes de la naturaleza humana, siempre corremos el riesgo de no realizar nuestra propia vida personal, la de

42 Gadamer, H. G., Truth and Method, Continuum, Londres-Nueva York, 2004, pp. 565-6.

43 Ética a Nicómaco, 1178a 5-9.

44 Ética a Nicómaco, 1178a 1.

45 Ética a Nicómaco, 1178b 34 y ss. 
cada cual, corremos el riesgo de vivir de modo alienado, de elegir la vida de (lo) otro. "Sería absurdo - escribe Aristóteles- que un hombre no eligiera su propia vida, sino la de otro ${ }^{46}$. Y esto puede ocurrir por imposición más o menos violenta, por obcecación, por capricho, por ignorancia, por falta de reflexión o escucha en medio de esta sociedad del ruido, o por falta de autocontrol, por mimetismo o por moda... ¡Son tantas las posibles causas de la inautenticidad! (Y si algo espera nuestra sociedad de los filósofos, por cierto, es que ayudemos a identificarlas).

En suma, todo el recorrido que hemos hecho nos orienta hacia un sentido de la vida que cumple con las condiciones buscadas. Es un sentido común, pues es concorde con la naturaleza humana, la que compartimos todos los miembros de la familia humana. Es un sentido de la vida personal, pues se concreta en la irrepetible diferencia constitutiva. Es también cognoscible, gracias a una gran pluralidad de fuentes, y razonable, pues responde ante el tribunal de la verdad práctica, lo que permite justificar razonablemente su elección ante uno mismo y ante los demás (y también ante Dios, añadiría el creyente).

\section{RESUMEN CONCLUSIVO}

Nos ha tocado vivir a cballo entre el fin de la modernidad y el comienzo de no sabemos todavía qué. Los tiempos modernos nos han aportado potentes instrumentos, pero nos han sumido en una cierta ignorancia del sentido. Nos cumple ahora investigar el sentido de la vida humana sin renunciar a los medios que hemos ganado durante la modernidad.

En esta clave hemos recuperado dos máximas antiguas en torno a las cuales parece haber un amplísimo consenso. Conforme a la primera, el sentido de cada vida está en la autorrealización. La segunda nos recuerda la necesidad de autoconocimiento, sin el cual la autorrealización sería imposible. Necesitamos, pues, un conocimiento, tanto de nuestra naturaleza humana, como de nuestra peculiar constitución personal.

A partir de aquí hemos recorrido muy someramente las actuales posiciones respecto de la naturaleza humana, y hemos apostado por una en concreto. Se trata de una teoría de la naturaleza humana muy próxima al sentido común y a la tradición aristotélica, según la cual somos animales sociales racionales. Hemos explorado, también muy superficialmente, estas tres características o diferencias propias de lo humano. En virtud de las mismas hemos descubierto que somos vulnerables, dependientes y autónomos. También sabemos que nuestro lugar propio está tanto en el entorno natural, tanto como entre la familia humana y en la esfera de lo espiritual. Todo ello nos da ya muchas orientaciones de vida. Pero, al fin y al cabo, mi vida es una vida personal, que tiene que realizarse también en función del individuo concreto que soy.

46 Ética a Nicómaco, 1178a 4-5. 
¿Podemos obtener conocimiento sobre esto?, ¿dicho conocimiento puede ser reputado como verdadero o falso sin relativismo? La respuesta nos viene dada a través de la noción de diferencia. La diferencia constitutiva es única en cada persona, en ella se integran todos los rasgos o diferencias, y, además, es formal, luego es, en principio, cognoscible. Ahora bien, para llegar a conocerla hace falta todo un arsenal muy variado de recursos, que incluye las ciencias y mucho más. Además, el tipo de verdad que podemos pedir a este conocimiento es la verdad práctica. A través del desarrollo de este concepto nos hemos dado cuenta de que en realidad el autoconocimiento (verdad) y la autorrealización (praxis) son procesos simultáneos y mutuamente dependientes que se retroalimentan.

Que para conocer a la persona y propiciar su realización hagan falta las ciencias naturales, sociales y humanas, además de las artes, la sabiduría religiosa y proverbial, la filosofía, el diálogo y la comunicación, la meditación, contemplación y reflexión, la praxis y la experiencia cotidiana, no es ciertamente un gran hallazgo. Es más bien una obviedad. Pero el objetivo aquí era precisamente el de presentar unas bases filosóficas que den cuenta de esta verdad obvia y que sean conformes a la misma.

Universidad de Valladolid

Alfredo Marcos

amarcos@fyl.uva.es

www.fyl.uva.es/ wfilosof/webMarcos

[Artículo aprobado para publicación en diciembre de 2016] 